\title{
A COMMENT ON THE ANALYSIS OF DATA GENERATED BY SIMULATION EXPERIMENTS* $\dagger$
}

\section{THOMAS H. NAYLOR $\ddagger$ AND THOMAS H. WONNACOTT§}

The purpose of this note is to suggest some improvements in current statistical analysis of computer-simulated data. To be concrete, we will take a recent paper by Rosser T. Nelson [7] which appears in this journal as the vehicle for our comments. In this article, Professor Nelson described a job-shop simulation experiment consisting of a three-factor incomplete factorial design. The purpose of the experiment was to evaluate the effects of four different labor force sizes, three different queue disciplines, and five different machine selection procedures on the mean time in system, the variance of the time in system, the maximum time in system, and the distribution function of time in system. A total of 36 runs were made out of a possible 60. (Nelson chose not to try all $4 \times 3 \times 5$ factor combinations.) Each run consisted of 32,000 jobs and on the basis of this sample he made the following inferences.

The mean and variance of the time in system for different queue disciplines exhibited a consistent pattern for both machine limited and labor and machine limited systems. The rank order of effectiveness of the queue disciplines with respect to the variance of time in system was FIFS, FCFS, SOT [7, p. 669].

Similar inferences were made about the effects of other factor combinations on the response variables.

* Received June 1967; revised January 1970.

† Supported by National Science Foundation Grants GS-1104 and GS-1926.

$\ddagger$ Duke University.

University of Western Ontario. 
If one is going to make inferences about differences in population means (or variances) or rankings of population means (or variances), isn't he obligated to say something about the degree of confidence which he places in these inferences? To make inferences of the type which Nelson has made without computing a single confidence interval is to cast serious doubts on the validity of his experiment.

If there is very little sampling fluctuation in the experimental results, then statistical analysis is a luxury. However, statistical analysis becomes almost a necessity in Nelson's experiment, where the sampling error is quite large. To see just how large the error is, we refer to his footnote on page 660 , where he considers the ratio $(\lambda)$ of variances of time in system for SOT compared to FCFS. In this footnote he shows that a sample of only 8,000 jobs would give an unstable estimate of the ratio $\lambda$. In fact, 4 such samples of 8,000 jobs each give $\lambda=1.12,1.52,0.99$ and $1.21-$ a clear indication of instability.

When these 4 samples are combined into one large sample of 32,000 jobs, the stability is improved ${ }^{1}$ but remains far from perfect. The combined sample of 32,000 jobs gives $\lambda=1.25$. The customary way to allow for the instability is by means of a $95 \%$ confidence interval, which turns out to be, ${ }^{2}$

$$
\text { true ratio }=1.25 \pm .35 \text {. }
$$

This means that if the computer generated unlimited data, we can say with $95 \%$ confidence that the true value which $\lambda$ would approach lies somewhere between .90 and 1.60. Such a wide confidence interval gives the reader a healthy respect for the limitations of computer simulation, and some insight as to why computer simulators so often disagree-statistical fluctuation. In fact, some readers might feel that the computer simulation is not a great improvement over common sense; for common sense suggests equally well that SOT yields a higher variance than the FCFS queue discipline.

Confidence intervals are only one of several statistical techniques that would be appropriate to analyze Professor Nelson's data. Several techniques are described in [6] and include (i) the $F$-test, (ii) multiple comparisons, and (iii) multiple ranking procedures.

(i) If one's experimental objective is to test the hypothesis that there is no difference between two or more population means, then the $F$-test is an appropriate analytical tool.

(ii) Typically, decision makers are interested not only in whether population means differ, but also in how much they differ. For example, how much do the population means associated with the different queue disciplines in Nelson's experiment differ? Multiple comparisons are the tools relevant to this query. They emphasize the use of confidence intervals rather than testing hypotheses.

(iii) If the objective of the experiment is to be reasonably sure of finding the best policy, second best policy, etc., then the best estimate of the ranking of a set of population means is the ranking of the sample means. Because of random error, however, sample rankings are quite likely to yield incorrect results, unless the sample size is adequate. (Professor Nelson ignored this fact.) To determine an adequate sample size to ensure a high probability of a correct ranking, multiple ranking procedures [3] are relevant.

1 The standard deviation of a sample mean based on 32,000 jobs is $\frac{1}{2}$ the standard deviation of a sample mean based on 8,000 jobs.

2 Although there are slightly better nonparametric methods available, we based our confidence interval on Student's t-distribution and used the logarithm of $\lambda$. 
Even more sophisticated techniques exist to solve more complicated problems of inference. To cite a few examples, spectral analysis [1] of time series can be used to help overcome the problem of serial correlation. Experimental designs $[2,4,5]$ facilitate the simultaneous analysis of multifactor simulation experiments.

In conclusion, we return to Professor Nelson's excellent idea of breaking the 32,000 runs into 4 subsamples of 8,000 each. The extent to which these 4 subsamples disagreed showed the sampling fluctuation, and provided the key to statistical inference about the true population parameters. The replication of 4 subsamples provided insight that is lost by taking one large sample and ignoring the scientific problem of reproducibility.

\section{References}

1. Frbmann, G. S., and Kiviat, P. J., "The Analysis of Simulation Generated Time Series," Management Science, Vol. 13, No. 7 (March 1967), pp. 525-557.

2. Hunter, J. S. ANd NayLor, Thomas H., "Experimental Designs for Computer Simulstion Experiments," Management Science, Vol. 16, No. 7 (March 1970), pp. 422-434.

3. Kleisnen, Jacx P., and Narlor, Thomas H., "The Ube of Multiple Ranking-Procedures to Analyze Simulations of Business and Economic Systems," Proceedings of the American Statistical Association, August 1969).

4. NatLos, Tromas H. (Editor), The Desion of Computer Simulation Experiments, Duke University Press, Durham, N. C. 1969.

5. - Computer Simulation Experiments with Models of Economic Systems, John Wiley \& Sons, New York, 1970.

6. - Wretz, Kennetr and Wonnacott, Thomas H., "Methods for Analyzing Data from Computer Simulation Experiments," Communications of the ACM, Vol. 10 (November 1967), pp. 703-710.

7. Netbon, Rosser T., "Labor and Machine Limited Production Systems," Management Science, Vol. 13, No. 9 (M8y 1967), pp. 648-671.

Reproduced with permission of the copyright owner. Further reproduction prohibited without permission. 